\title{
Whether noninvasive optimization of AV and VV delays improves the response to cardiac resynchronization therapy
}

\author{
Bożena Urbanek, Michał Chudzik, Artur Klimczak, Marcin Rosiak, \\ Joanna Lewek, Jerzy Krzysztof Wranicz \\ Department of Electrocardiology, Medical University of Lodz, Lodz, Poland
}

\begin{abstract}
Background: Device optimization is not routinely performed in patients who underwent cardiac resynchronization therapy (CRT) device implantation. Noninvasive optimization of CRT devices by measurement of cardiac output (CO) can be used as a simple method to assess ventricular systolic performance. The aim of this study was to assess whether optimization of atrioventricular $(A V)$ and interventricular $(V V)$ delay can improve hemodynamic response to CRT and whether this optimization should be performed for each patient individually.

Methods: Twenty patients with advanced heart failure New York Heart Association (NYHA) class III/IV, left ventricular ejection fraction $\leq 35 \%$ and left bundle branch block $(Q R S \geq 120 \mathrm{~ms})$ in sinus rhythm were evaluated from $24 \mathrm{~h}$ to $48 \mathrm{~h}$ after implantation of a CRT device by means of impedance cardiography (ICG). CO was first measured at each patient's intrinsic rhythm. Patients then underwent adjustments of $A V$ and $V V$ delay from $80 \mathrm{~ms}$ to $140 \mathrm{~ms}$ and from $-60 \mathrm{~ms}$ to $+60 \mathrm{~ms}$, respectively in $20 \mathrm{~ms}$ increment steps and CO at each setting was measured by ICG. Both AV and VV delays were programmed according to the greatest improvement in CO compared to intrinsic rhythm.

Results: There was a statistically significant increase in $\mathrm{CO}$ measured at the intrinsic rhythm compared to different $A V$ delay by mean of $21 \%(3.8 \pm 1.0 \mathrm{vs} .4 .6 \pm 0.1 \mathrm{~L} / \mathrm{min}, p<0.05)$. Optimal AV/VV delays with left ventricle-preexcitation or simultaneous biventricular pacing caused additional increased CO from intrinsic rhythm by mean of $32.6 \%$ (3.8 \pm 1.0 vs. $5.04 \pm$ $\pm 1.0 \mathrm{~L} / \mathrm{min}, p<0.05)$. Optimal AV/VV setting delays also resulted in improved hemodynamic responses compared to $V V$ factory setting delay.

Conclusions: Both AV and VV delay optimization should be performed in clinical practice. Optimal AV delay improved outcome. However, combination of optimized AV/VV delays provided the best hemodynamic response. Optimized AV/VV delays with left ventricle-preexcitation or simultaneous biventricular pacing increased hemodynamic output compared to intrinsic rhythm and VV factory setting delay. (Cardiol J 2013; 20, 4: 411-417)
\end{abstract}

Key words: cardiac resynchronization therapy, noninvasive optimization, impedance cardiography

Address for correspondence: Bożena Urbanek, PhD, Department of Electrocardiology, Medical University of Lodz, ul. Sterlinga 1/3, 91-425 Łódź, Poland, e-mail: bozena_urbanek@op.pl 


\section{Introduction}

Cardiac resynchronization therapy (CRT) is an important therapeutic option for patients with advanced heart failure (when optimal pharmacotherapy is insufficient) [1]. The essence of CRT is to increase cardiac output (CO), reducing interventricular (VV) conduction delay caused by left bundle branch block [2]. CRT reduces clinical symptoms of heart failure, decreasing incidence of hospitalization, and also significantly decreasing mortality in these patients [3, 4]. The success of CRT depends on many factors such as appropriate location of the left ventricular (LV) electrode, and optimal timing of atrioventricular (AV) and VV delays [5, 6]. Optimal programming of $\mathrm{AV}$ and $\mathrm{VV}$ delays plays an important role in reducing the proportion of patients who derive no apparent benefit from CRT (the so called "non-responders"), despite correct positioning of the LV electrode [7]. Timing of optimal AV and VV conduction differs among patients and requires individual settings. The most common non-invasive methods used in optimization of CRT settings are echocardiography and impedance cardiography (ICG).The purpose of this study was to see whether optimization of $\mathrm{AV}$ and $\mathrm{VV}$ delays by ICG contributes to improvement of hemodynamic response to CRT and whether this optimization should be performed for each patient individually.

\section{Methods}

\section{Patient characteristics}

The study included 20 patients with advanced heart failure, scheduled for implanting a CRT device. All the patients were of New York Heart Association (NYHA) class III heart failure, despite appropriate medical therapy, with impaired $\mathrm{LV}$ ejection fraction (LVEF) $\leq 35 \%$, wide QRS due to left bundle branch block (QRS duration $\geq 120 \mathrm{~ms}$ ) and sinus rhythm on 12-lead surface electrocardiography. For all the patients echocardiography was performed, measurements of LV dimensions, LVEF, extent of interventricular mechanical dyssynchrony, and evaluation for valve disease, particularly mitral regurgitation were obtained. All the patients were receiving optimal medical treatment. Baseline characteristics of the study patients are detailed in Table 1 .

The present study was approved by the Institutional Review Board of the Medical University of Lodz. The study protocol was approved by the local Ethics Committee, and informed consent was obtained from all the patients.
Table 1. Patients' characteristics.

\begin{tabular}{|c|c|}
\hline Characteristic & $\begin{array}{c}\text { Value (total } \\
\text { cohort: } n=20 \text { ) }\end{array}$ \\
\hline Age [years] & $\begin{array}{c}66.9 \pm 7.6 \\
\text { (range 55-78) }\end{array}$ \\
\hline Gender (female/male) & $2 / 18$ \\
\hline \multicolumn{2}{|l|}{ Etiology } \\
\hline Nonischemic & $7(35 \%)$ \\
\hline QRS [ms] & $\begin{array}{c}176 \pm 27 \\
\text { (range 120-220) }\end{array}$ \\
\hline $\mathrm{PQ}[\mathrm{ms}]$ & $172 \pm 31$ \\
\hline LVEDV [mL] & $251 \pm 71$ \\
\hline LVESV [mL] & $186 \pm 62$ \\
\hline LVEF [\%] & $\begin{array}{c}25 \pm 4.7 \\
\text { (range 19-35) }\end{array}$ \\
\hline VV mechanical delay [ms] & $50 \pm 30$ \\
\hline NYHA class III & $20(100 \%)$ \\
\hline ACEI & $19(95 \%)$ \\
\hline Beta-blockers & $17(85 \%)$ \\
\hline Spironolactone & $11(55 \%)$ \\
\hline Loop diuretics & $20(100 \%)$ \\
\hline
\end{tabular}

\section{Implantation}

A CRT defibrillator (CRT-D) was implanted in all patients except one who received a CRT pacemaker (CRT-P). The implantation was performed according to routine procedure in all patients. The LV lead was positioned via the over-the-wire technique in the coronary sinus vein branch that was the easiest. Next impedance measurements were performed in this position by ICG. We then positioned the LV lead at 2 to 4 different sites, depending on the anatomy of the coronary sinus vein branches. Hemodynamic measurements were obtained at each site, in order to obtain the best response. The right atrial lead was implanted in the right atrial appendage and finally the right ventricular (RV) lead was placed in the midseptum or RV apex according to operator preference. For all patients the device was programmed in DDD mode with a lower pacing rate at $40 \mathrm{bpm}$ to $50 \mathrm{bpm}$ to ensure continuous atrial synchronous biventricular tracking of the intrinsic sinus rhythm [8]. The AV delay was set at $120 \mathrm{~ms}$ as a standard value [9], with VV delay set at 0 ms (VV factory setting delay). These parameters were unchanged until optimization. 


\section{Impedance cardiography}

ICG was performed with a commercial noninvasive continuous $\mathrm{CO}$ system (CardioScreen; Medizinische Messtechnik GmbH, Ilmenau, Germany) used for noninvasive hemodynamic measurements and monitoring of hemodynamic parameters.

Changes in volume and velocity of blood in the aorta cause variations in the thoracic bio-impedance which is measured and displayed as the ICG waveform. With each cardiac cycle, thoracic fluid volume changes, which affects the impedance to the electrical signal transmitted by the sensors. This signal is applied to the innovative Physiological Adaptive Signal Analysis algorithm to provide key hemodynamic parameters noninvasively and continuously [10]. The ICG system was composed of a high-frequency $(85 \mathrm{kHz})$ sine wave generator with 4 dual electrodes placed on either side of the body, which allowed for noninvasive measurement of $\mathrm{CO}$ and other hemodynamic parameters calculated on a beat-to-beat basis from the transthoracic impedance signal.

\section{Optimization protocol}

Device settings were optimized within $24 \mathrm{~h}$ to $48 \mathrm{~h}$ after CRT device implant via ICG. Patients were examined in the supine position in a silent room to reduce the impact of sympathetic activation by external stimuli. Before optimization each patient rested for a period of stabilization and equilibration (10 min) followed by $\mathrm{AV}$ and then $\mathrm{VV}$ delay optimization. The DDD mode, with a lower rate limit of $40 \mathrm{bpm}$ to avoid effects of atrial pacing on the AV interval, was programmed for each patient [8]. Telemetry between the CRT device and the programmer was disconnected during data acquisition, at each parameter setting, to avoid interference. The period for each AV and VV delay setting was preceded by a stabilization period of $1 \mathrm{~min}$ and at each stage measurements were made 4 times, with 20 cycles of beat for each reading and the average of all readings was calculated. The first stage of the optimization protocol was the $\mathrm{CO}$ measurement with each patient's intrinsic rhythm. With the VV fixed at $0 \mathrm{~ms}$, AV delay optimization was then adjusted from $80 \mathrm{~ms}$ to $140 \mathrm{~ms}$ in $20 \mathrm{~ms}$ increment steps and the $\mathrm{CO}$ was registered. $\mathrm{AV}$ delay values longer than $140 \mathrm{~ms}$ were not analyzed because of correct native AV conduction. The AV delay was set according to the largest $\mathrm{CO}$ (optimal AV delay). Next, with the optimized AV delay, opti- mal VV delay was estimated, and $\mathrm{CO}$ at each setting was measured (AV/VV delays). The VV delays were adjusted in $20 \mathrm{~ms}$ intervals through a set from LV-preexcitation by $-60 \mathrm{~ms}$ to RV-preexcitation by $+60 \mathrm{~ms}$, according to the previously published methods [11]. The optimal device setting was a combination of $\mathrm{AV}$ and $\mathrm{VV}$ delays providing the largest $\mathrm{CO}$ (optimal AV/VV delays). In addition, CO at the VV factory setting delay was compared to intrinsic rhythm and optimal AV/VV delays.

\section{Statistical analysis}

Statistical analyses were performed with STATISTICA PL 9.0 software. Numerical variables are presented as mean \pm standard deviation (SD). For categorical variables the number of observations (n) and percentage (\%) are presented. Normality was tested with the t test for dependent samples and the Shapiro-Wilk test for normality. Analysis of variance (ANOVA) with repeated measures and post-hoc analysis was used to compare numerical variables. Results were considered statistically significant for $\mathrm{p}<0.05$.

\section{Results}

\section{Lead placement}

The individual best hemodynamic response for each patient was associated with midlateral placement of the LV lead in 9 patients, apicolateral placement in 4 patients, basolateral placement in 3 patients, apicoposterior placement in 2 patients and basal-anterior and basal-posterior placement in 1 patient. The RV lead was positioned at RV apex in $14(70 \%)$ patients and at RV midseptum in $6(30 \%)$ patients.

\section{Setting of optimal AV and VV delays}

Using the noninvasive continuous $\mathrm{CO}$ system an optimal AV delay setting of $80 \mathrm{~ms}$ was measured for $1(5 \%)$ patient, $100 \mathrm{~ms}$ for $6(30 \%)$ patients, $120 \mathrm{~ms}$ for 7 (35\%) patients and $140 \mathrm{~ms}$ for $6(30 \%)$ patients. The mean optimal AV delay value was $118 \pm 18 \mathrm{~ms}$. The most common optimal VV delay value was programmed with simultaneous biventricular pacing $(0 \mathrm{~ms})$ for $9(45 \%)$ patients, and with preexcitation of $\mathrm{LV}$ pacing by $-40 \mathrm{~ms}$ for 7 (35\%) patients, by $-60 \mathrm{~ms}$ for $3(15 \%)$ patients and by $-20 \mathrm{~ms}$ for $1(5 \%)$ patient. There were no patients with RV preexcitation. The mean optimal VV delay was $-23 \pm 23 \mathrm{~ms}$. Programmed settings for optimal $\mathrm{AV}$ and VV delays are shown in Figure 1. 


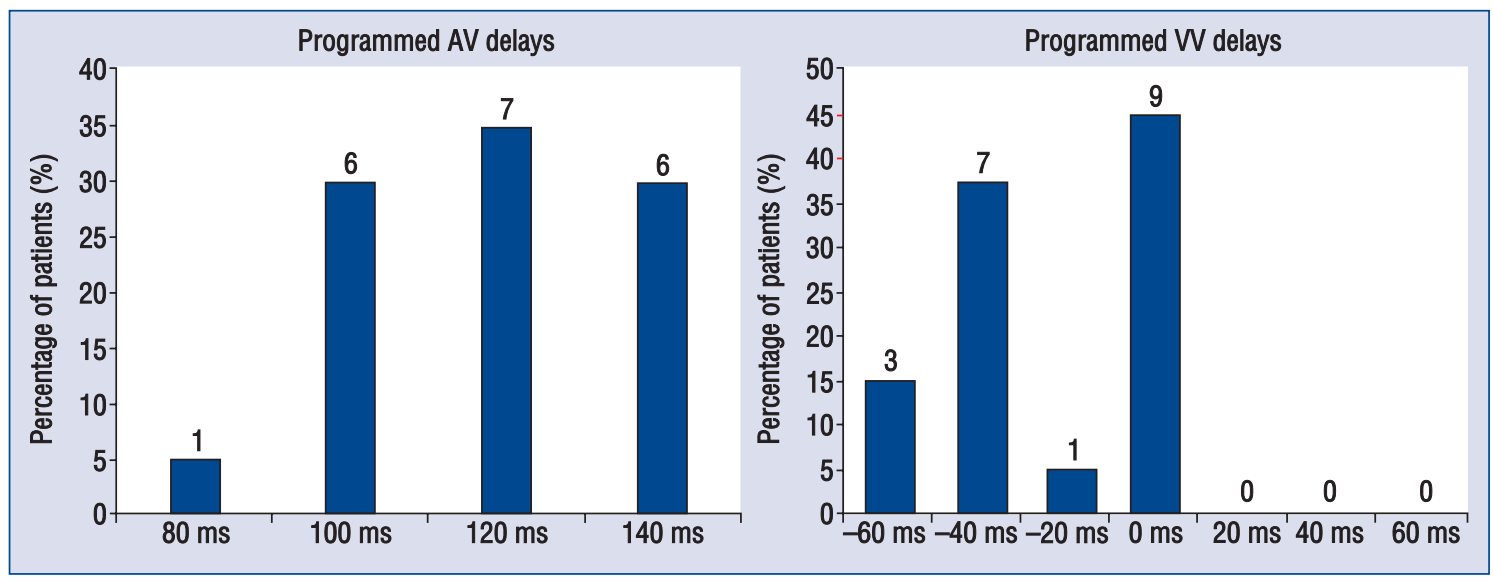

Figure 1. The frequency of different settings of optimal AV and VV delays.

\section{Outcome of noninvasive $\mathrm{CO}$ measured with optimal AV and VV delay settings}

The $\mathrm{CO}$ value obtained at intrinsic rhythm was $3.8 \pm 1.0 \mathrm{~L} / \mathrm{min}$. The $\mathrm{CO}$ obtained at intrinsic rhythm compared with the different AV delay was increased by $21 \%(3.8 \pm 1.0$ vs. $4.6 \pm 0.1 \mathrm{~L} / \mathrm{min}$, $\mathrm{p}<0.05)$. The largest $\mathrm{CO}(4.7 \mathrm{~L} / \mathrm{min}$, increase of $23 \%$ when compared to intrinsic rhythm, $\mathrm{p}<0.05$ ) was achieved when AV delay was set at $120 \mathrm{~ms}$ (Fig. 2).

There was a significant increase in $\mathrm{CO}$ with all $\mathrm{AV}$ delays compared to $\mathrm{CO}$ measured at intrinsic rhythm. No significant differences were observed between $\mathrm{CO}$ values measured with different $\mathrm{AV}$ delays (Table 2).

With different $\mathrm{AV} / \mathrm{VV}$ delays optimization $\mathrm{CO}$ improved from intrinsic rhythm by $18.4 \%$ (3.8 \pm \pm 1.0 vs. $4.5 \pm 0.2 \mathrm{~L} / \mathrm{min}, \mathrm{p}<0.05)$. The largest improvement in $\mathrm{CO}$ of $24.4 \%$ (3.8 \pm 1.0 vs. $4.7 \pm 0.9, \mathrm{p}<0.05 \mathrm{~L} / \mathrm{min}$ ) was obtained with AV/VV delays set up at simultaneous biventricular pacing (Fig. 3). There was a significant increase in $\mathrm{CO}$ for all VV delays compared to $\mathrm{CO}$ at intrinsic rhythm. Pacing with LV preexcitation by $-60 \mathrm{~ms}$ and by

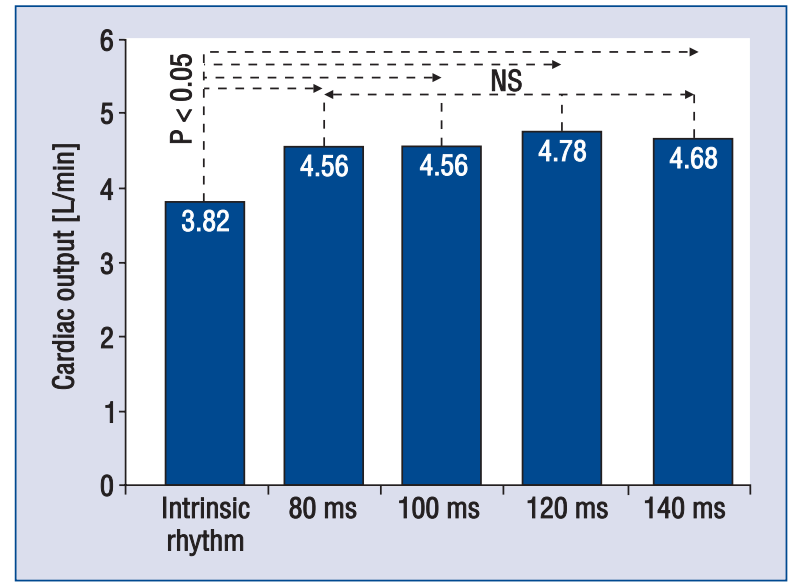

Figure 2. The effect on cardiac output in different AV delay settings.

$-40 \mathrm{~ms}$ showed significant differences in $\mathrm{CO}$ compared to RV preexcitation (Table 3 ).

The mean optimal AV/VV delays resulted in an increase of $\mathrm{CO}$ from baseline by $32.6 \%$ (3.8 \pm \pm 1.0 vs. $5.04 \pm 1.0 \mathrm{~L} / \mathrm{min}, \mathrm{p}<0.05)$. In additional

Table 2. Statistical value for cardiac output with different AV delay settings.

\begin{tabular}{lccccc}
\hline Variable (CO/AV) & Intrinsic rhythm & $\mathbf{8 0 ~} \mathbf{~ m s}$ & $\mathbf{1 0 0} \mathbf{~ m s}$ & $\mathbf{1 2 0} \mathbf{~ m s}$ & $\mathbf{1 4 0} \mathbf{~ m s}$ \\
\hline Intrinsic rhythm & $\mathrm{x}$ & $\mathrm{p}<0.05$ & $\mathrm{p}<0.05$ & $\mathrm{p}<0.05$ & $\mathrm{p}<0.05$ \\
$80 \mathrm{~ms}$ & $\mathrm{p}<0.05$ & $\mathrm{x}$ & $1.000^{*}$ & $0.514^{*}$ & $0.910^{*}$ \\
$100 \mathrm{~ms}$ & $\mathrm{p}<0.05$ & $1.000^{*}$ & $\mathrm{x}$ & $0.537^{*}$ & $0.921^{*}$ \\
$120 \mathrm{~ms}$ & $\mathrm{p}<0.05$ & $0.514^{*}$ & $0.537^{*}$ & $\mathrm{x}$ & $0.951^{*}$ \\
$140 \mathrm{~ms}$ & $\mathrm{p}<0.05$ & $0.910^{*}$ & $0.921^{*}$ & $0.951^{*}$ & $\mathrm{x}$ \\
\hline
\end{tabular}

$\mathrm{CO}$ - cardiac output; AV — atrioventricular delay; ${ }^{*} \mathrm{p}$ value not significant 


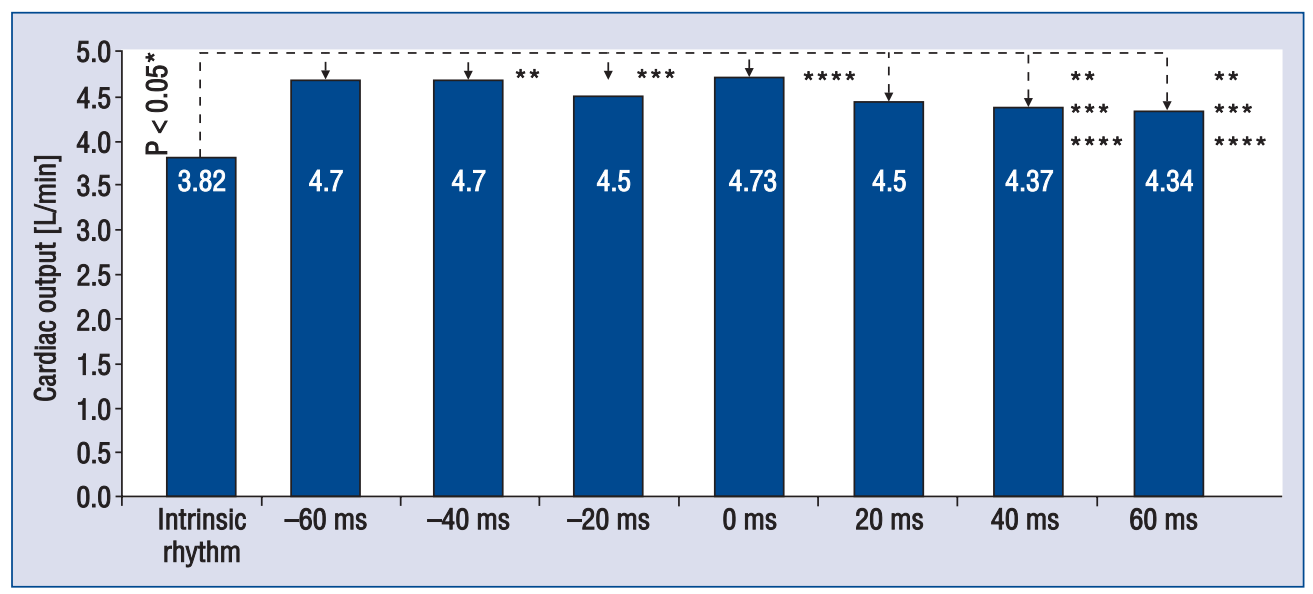

Figure 3. The effect on cardiac output in different AV/VV delay settings; *intrinsic rhythm cardiac output was statistically different from other values; ${ }^{* *}-60 \mathrm{~ms}$ was statistically significant compared to $40 \mathrm{~ms}$ and $60 \mathrm{~ms} ;{ }^{* *}-40 \mathrm{~ms}$ was statistically significant compared to $40 \mathrm{~ms}$ and $60 \mathrm{~ms} ;{ }^{* * *} 0 \mathrm{~ms}$ was statistically significant compared to $40 \mathrm{~ms}$ and $60 \mathrm{~ms}$.

Table 3. Statistical value of cardiac output with different AV/VV delay settings.

\begin{tabular}{|c|c|c|c|c|c|c|c|c|}
\hline Variable (CO/VV) & $\begin{array}{l}\text { Intrinsic } \\
\text { rhythm }\end{array}$ & $-60 \mathrm{~ms}$ & $-40 \mathrm{~ms}$ & $-20 \mathrm{~ms}$ & $0 \mathrm{~ms}$ & $20 \mathrm{~ms}$ & $40 \mathrm{~ms}$ & $60 \mathrm{~ms}$ \\
\hline Intrinsic rhythm & & $p<0.05$ & $p<0.05$ & $p<0.05$ & $p<0.05$ & $p<0.05$ & $p<0.05$ & $p<0.05$ \\
\hline$-60 \mathrm{~ms}$ & & & $1.000^{*}$ & $0.598 *$ & $1.000^{*}$ & $0.299 *$ & $p<0.05$ & $p<0.05$ \\
\hline$-40 \mathrm{~ms}$ & & & & $0.598^{*}$ & $1.000^{*}$ & $0.299 *$ & $p<0.05$ & $p<0.05$ \\
\hline$-20 \mathrm{~ms}$ & & & & & $0.410^{*}$ & $0.999 *$ & $0.935^{*}$ & $0.848^{*}$ \\
\hline $0 \mathrm{~ms}$ & & & & & & $0.169 *$ & $p<0.05$ & $p<0.05$ \\
\hline $20 \mathrm{~ms}$ & & & & & & & $0.996^{*}$ & $0.979 *$ \\
\hline $40 \mathrm{~ms}$ & & & & & & & & $1.000^{*}$ \\
\hline $60 \mathrm{~ms}$ & & & & & & & & \\
\hline
\end{tabular}

$\mathrm{CO}$ - cardiac output; AV — atrioventricular delay; *p value not significant

CO during pacing with the VV factory setting delay was lower compared to mean optimal AV/VV delays by $7.2 \%(4.7 \pm 0.9$ vs. $5.04 \pm 1.0 \mathrm{~L} / \mathrm{min}, \mathrm{p}<0.05)$. A comparison of $\mathrm{CO}$ at intrinsic rhythm with the VV factory setting delay, and with optimal AV/VV delay settings is shown in Figure 4.

There was no significant difference in heart rate during CO estimated at baseline $(69 \pm 7.5 \mathrm{bpm})$, with AV delay optimization $(70 \pm 5.3 \mathrm{bpm})$, with the VV delay factory setting $(70 \pm 2.5 \mathrm{bpm})$ or with AV/VV delay optimization $(69.4 \pm 7 \mathrm{bpm}$, $\mathrm{p}>0.05)$.

\section{Discussion}

Invasive monitoring of hemodynamics in heart failure has been the gold standard, but it still

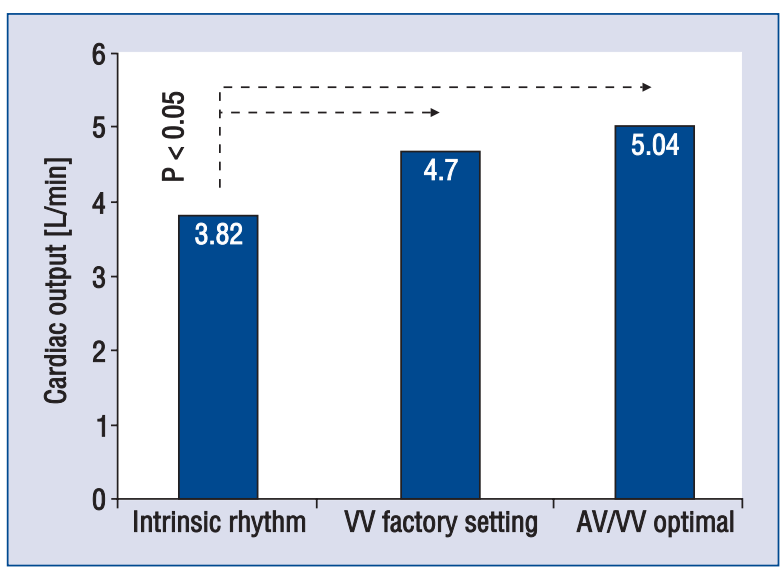

Figure 4. Comparison of cardiac output changes in intrinsic rhythm, VV factory setting and optimal AV/VV delays. 
carries significant associated risks. Recent studies have described the use of ICG to guide the optimal setting of CRT devices. Use of this technique has resulted in significant improvements in $\mathrm{CO}$, owing to adjustment of both $\mathrm{AV}$ and $\mathrm{VV}$ delays [10, 11]. Result of the present study confirmed that noninvasive ICG is a useful and reliable method for optimizing AV and VV delays. There were significant variations in the optimal CRT timing settings between patients. Therefore, to achieve benefit from CRT, it is necessary to optimize each patient's device individually not to use "universal" settings for all. The present results demonstrated that individual adjustment of AV and VV delays, compared to intrinsic rhythm, resulted in hemodynamic improvements. An individually based approach to the largest $\mathrm{CO}$ was associated with different $\mathrm{AV}$ delays and VV delays with LV preexcitation or simultaneous biventricular pacing.

\section{AV delay}

The setting of AV delay for individual patients measured by echocardiography allows for electromechanical synchrony of atrial and ventricular contractions. In addition, it improves cardiac contractile function and therefore improves long-term prognosis [12]. As a quick and consistent measurement, $\mathrm{CO}$ is the most frequently used hemodynamic parameter for finding an optimal AV delay. Early studies have shown that compared to the factory setting a properly adjusted AV delay can contribute to CO increases up to $40 \%$ [13]. The development of ICG has provided the opportunity to measure $\mathrm{CO}$ noninvasively in contrast to previous invasive techniques. Results received with ICG correlate closely with those of invasive techniques $[14,15]$. Studies have also demonstrated that optimization of AV delay by ICG is a useful and less expensive alternative to echocardiography during standard dual chamber pacing $[16,17]$. Another study demonstrated that ICG can be used to determine $\mathrm{CO}$ during $\mathrm{LV}$ pacing at various $\mathrm{AV}$ intervals, with strong correlation to results obtained by Doppler echocardiography [18]. In addition, hemodynamic benefits of optimization required individual adjustment of AV delay. Our present results are consistent with these observations.

\section{VV delay}

Apart from benefits of AV delay manipulation, modification of VV delay might also improve the hemodynamic response to CRT. Some invasive studies assessing the hemodynamic response to VV programming have demonstrated that se- quential biventricular pacing is more effective than simultaneous biventricular pacing [19, 20]. Similar findings were reported by Marsan et al. [21]. They optimized sequential biventricular pacing which, increased LV systolic performance (EF and LV outflow-tract- velocity-time-integral) compared to simultaneous stimulation estimated by noninvasive Doppler echocardiography. Other noninvasive studies have confirmed that a better effect on hemodynamic response in CRT is obtained with both AV and VV delays as verified by 3-dimensional echocardiography [22], radionuclide ventriculography [23], finger plethysmography [24] and recently, ICG $[10,11,25]$. Comparing optimization techniques, echocardiographic methods yield statistically insignificant data in the majority of patients $(62-82 \%)$ whereas ICG yields statistically significant results in $84 \%$ and $75 \%$ of patients for AV and VV interval optimization respectively [26]. It seems that ICG is free of some of the limitations of echocardiography. It is noninvasive, replicable, undemanding of qualified personnel, and less expensive than echocardiography [16]. In clinical practice, individual adjustment of $\mathrm{VV}$ delay is often ignored [27]. This might be due to the fact that VV delay optimization based on echocardiography is a relatively time-consuming procedure.

With a considerable growth in CRT implant, it will be impossible to optimize devices echocardiographically in all patients [28]. The study of Bogaard and associates reported that the optimal VV delay is $-30 \mathrm{~ms}$ ( $-60 \mathrm{~ms}$ to $5 \mathrm{~ms}$ ) and $-20 \mathrm{~ms}$ ( $-40 \mathrm{~ms}$ to $0 \mathrm{~ms}$ ) at non-optimal and optimal LV lead sites, respectively [29]. Heinroth and associates compared VV delays and reported that LV preexcitation by $40 \mathrm{~ms}$ resulted the largest mean $\mathrm{CO}$, and similar values were obtained with LV preexcitation by $20 \mathrm{~ms}$ and $60 \mathrm{~ms}$ [10]. The optimal pacing set-up varied greatly from patient to patient. In present study, optimization of both $\mathrm{AV}$ and $\mathrm{VV}$ delays by ICG turned out simply and easy method, confirming the efficacy of CRT setting optimization. The greatest benefit for patients with individually optimized AV delay were obtained with VV delay and $\mathrm{LV}$ preexcitation by $-60 \mathrm{~ms}$ and by $-40 \mathrm{~ms}$; the mean optimal VV delay with improvement hemodynamic response was $-23 \pm 23 \mathrm{~ms}$. Optimal settings differed from patient to patient.

\section{Limitations of the study}

The present study was based on a small nonrandomized population. According to the fact that there are studies reporting that optimal AV delay values during rest or exercise do not differ signi- 
ficantly [30], we performed AV and VV optimization with patients in the supine position at rest. Nonetheless, it would be worthwhile to assess AV and VV delays under other conditions, for example during normal daily activity or exercise [30]. Finally our optimization protocol was based on previous study [11]. We optimized AV delays during simultaneous biventricular pacing and then optimized VV delays according to the optimal AV delay.

\section{Conclusions}

Both AV and VV delay optimization should be performed in clinical practice. Echocardiographic techniques, despite being time-consuming, deliver practical information. However, it appears that other methods of assessment might also be beneficial. AV and VV delay optimization by ICG is a valuable method and an interesting alterative. Optimal AV delay improved $\mathrm{CO}$ in the present study. However, combination of optimized AV/VV delays provided the best hemodynamic response. Compared to intrinsic rhythm and VV factory setting delay optimal AV/VV delays with LV-preexcitation and simultaneous biventricular pacing increased hemodynamic output. The ICG is a useful noninvasive technique for optimization of CRT.

\section{Conflict of interest: none declared}

\section{References}

1. Vardas PE, Auricchio A, Blanc JJ et al. Guidelines for cardiac pacing and cardiac resynchronization therapy. Eur Heart J, 2007; 28: 2256-2295

2. Cohen TJ, Klein J. Cardiac resynchronization therapy for treatment of chronic heart failure. J Invasive Cardiol, 2002; 14: 48-53.

3. Cazeau S, Leclercq C, Lavergne T et al. Effects of multisite biventricular pacing in patients with heart failure and intraventricular conduction delay. N Engl J Med, 2001; 344: 873-880.

4. Salukhe TV, Dimopoulos K, Francis D. Cardiac resynchronisation may reduce all-cause mortality: meta-analysis of preliminary COMPANION data with CONTAK-CD, InSync ICD, MIRACLE and MUSTIC. Int J Cardiol, 2004; 93: 101-103.

5. Gold MR, Auricchio A, Hummel JD et al. Comparison of stimulation sites within left ventricular veins on the acute hemodynamic effects of cardiac resynchronization therapy. Heart Rhythm, 2005; 2: 376-381.

6. Auricchio A, Ding J, Spinelli JC et al. Cardiac resynchronization therapy restores optimal atrioventricular mechanical timing in heart failure patients with ventricular conduction delay. J Am Coll Cardiol, 2002; 39: 1163-1169.

7. Birnie DH, Tang AS. The problem of non-response to cardiac resynchronization therapy. Curr Opin Cardiol, 2006; 21: 20-26.

8. Bernheim A, Ammann P, Sticherling C et al. Right atrial pacing impairs cardiac function during resynchronization therapy: Acute effects of DDD pacing compared to VDD pacing. J Am Coll Cardiol, 2005; 45: 1482-1487.

9. Braun MU, Schnabel A, Rauwolf T, Schulze M, Strasser RH. Impedance cardiography as a noninvasive technique for atrioventricular interval optimization in cardiac resynchronization therapy. J Interv Card Electrophysiol, 2005; 13: 223-229.
10. Heinroth KM, Elster M, Nuding S et al. Impedance cardiography: A useful and reliable tool in optimization of cardiac resynchronization devices. Europace, 2007; 9: 744-750.

11. Khan FZ, Virdee MS, Pugh PJ, Read PA, Fynn SP, Dutka DP. Non-invasive cardiac output measurements based on bioreactance for optimization of atrio- and interventricular delays. Europace, 2009; 11: 1666-1674.

12. Morales MA, Startari U, Panchetti L, Rossi A, Piacenti M. Atrioventricular delay optimization by doppler-derived left ventricular $\mathrm{dP} / \mathrm{dt}$ improves 6 -month outcome of resynchronized patients. Pacing Clin Electrophysiol, 2006; 29: 564-568.

13. Ovsyshcher IE. Toward physiological pacing: optimization of cardiac. Pacing Clin Electrophysiol, 1997; 20: 861-865.

14. Padeletti L, Porciani MC, Ritter Pet al. Atrioventricular interval optimization in the right atrial appendage and interatrial septum pacing: a comparison between echo and peak endocardial acceleration measurements. Pacing Clin Electrophysiol, 2000; 23: 1618-1620.

15. Rosenberg P, Yancy CW. Noninvasive assessment of hemodynamics: an emphasis on bioimpedance cardiography. Curr Opin Cardiol, 2000; 15: 151-155.

16. Ovsyshcher I, Furman S. Impedance cardiography for cardiac output estimation in pacemaker patients. PACE, 1993; 16: 1412-1422.

17. Kindermann M, Fröhlig G, Doerr T, Schieffer H. Optimizing the $\mathrm{AV}$ delay in DDD pacemaker patients with high degree AV block: Mitral valve Doppler versus impedance cardiography. PACE, 1997; 20: 2453-2462.

18. Tse HF, Yu C, Park E, Lau CP. Impedance cardiography for atrioventricular interval optimization during permanent left ventricular pacing. Pacing Clin Electrophysiol, 2003; 26: 189-191.

19. Perego GB, Chianca R, Facchini M et al. Simultaneous vs. sequential biventricular pacing in dilated cardiomyopathy: An acute hemodynamic study. Eur J Heart Fail, 2003; 5: 305-313.

20. van Gelder BM, Bracke FA, Meijer A, Lakerverd LJ, Pijls NH. Effect of optimizing the VV interval on left ventricular contractility in cardiac resynchronization therapy. Am J Cardiol, 2004; 93: $1500-1503$.

21. Marsan NA, Bleeker GB, van Bommel RJ, Borleffs JW, Holman ER. Cardiac resynchronization therapy in patients with ischemic versus non-ischemic heart failure: Differential effect of optimizing interventricular pacing interval. Am Heart J, 2009; 158: 769-776.

22. Sogaard P, Egeblad H, Pedersen AK et al. Sequential versus simultaneous biventricular resynchronization for severe heart failure: Evaluation by tissue Doppler imaging. Circulation, 2002; 106: 2078-2084.

23. Burri $\mathrm{H}$, Sunthorn $\mathrm{H}$, Somsen A et al. Optimizing sequential biventricular pacing using radionuclide ventriculography. Heart Rhythm, 2005; 2: 960-965.

24. Butter C, Stellbrink C, Belalcazar A et al. Cardiac resynchronization therapy optimization by finger plethysmography. Heart Rhythm, 2004; 1: 568-575.

25. Khan FZ, Virdee MS, Hutchinson J et al. Cardiac resynchronization therapy optimization using noninvasive cardiac output measurement. Pacing Clin Electrophysiol, 2011; 34: 1527-1536.

26. Turcott RG, Witteles RM, Wang PJ, Vagelos RH, Fowler MB, Ashley EA. Measurement precision in the optimization of cardiac resynchronization therapy. Circ Heart Fail, 2010; 3: 395-404.

27. Gras D, Gupta MS, Boulogne E, Guzzo L, Abraham WT. Optimization of $\mathrm{AV}$ and $\mathrm{VV}$ delays in the real-world CRT patient population: an international survey on current clinical practice. Pacing Clin Electrophysiol, 2009; 32 (suppl. 1): S236-S239.

28. Ypenburg C, Van de Veire N, Westenberg JJ et al. Noninvasive imaging in cardiac resynchronization therapy: Part 2: Follow-up and optimization of settings. Pacing Clin Electrophysiol, 2008; 31: 1628-1639.

29. Bogaard MD, Doevendans PA, Leenders GE al. Can optimization of pacing settings compensate for a non-optimal left ventricular pacing site? Europace, 2010; 12: 1262-1269.

30. Prochnau D, Forberg T, Kühnert H, Heineke M, Figulla HR, Surber R. Optimization of the atrioventricular delay during cardiac resynchronization therapy using a device for non-invasive measurement of cardiac index at rest and during exercise. Europace, 2012; 14: 249-253. 